\title{
Endocrine complications of immunotherapies: a review
}

\author{
Authors: Rosie Hattersley, ${ }^{A}$ Melanie Nana ${ }^{B}$ and Andrew J Lansdown ${ }^{C}$
}

Use of immune checkpoint inhibitors in cancer treatment has increased vastly over the past decade, as both single and combination agent therapies. While having a positive impact on survival rates, adverse effects have been noted, with endocrine effects in around $10 \%$ of patients. Thyroid disease and hypophysitis are the most commonly encountered, with diabetes mellitus and primary adrenal insufficiency also reported, as well as more rare endocrinopathies. Patient and clinician education to raise awareness of these effects, as well as regular monitoring to enable early recognition, diagnosis and prompt treatment of the immune side effects, are key. In this review, we discuss the aetiology, presentation and management of the endocrine complications of immunotherapies that are relevant to the general physician, as well as highlighting important areas where further research is still needed.

KEYWORDS: immunotherapy, cancer, endocrine, thyroid, hypophysitis

\section{DOI: $10.7861 /$ clinmed.2020-0827}

\section{Introduction}

The advent of immunotherapy has revolutionised cancer treatment across the world. Through harnessing the immune system to target malignant cells, previously untreatable cancers now have radically improved survival rates. ${ }^{1}$ The use of immunotherapy has increased over the past decade, becoming commonly used for a number of solid tumours and haematological malignancies. ${ }^{2}$ However, a predicted $60-95 \%$ of patients experience ill effects, which, if left untreated, may be life-threatening. ${ }^{3,4}$

Checkpoint inhibitors are a group of monoclonal antibody therapies that can be used in isolation or in combination. They are given via intravenous infusion, typically every 3-6 weeks, for up to 2 years. They exert their effect by upregulating the immune response to malignant cells, blocking the usual inhibitory pathways of T-cell regulation. ${ }^{5}$ The receptors cytotoxic T-lymphocyte antigen 4 (CTLA-4) and programmed cell death protein 1 (PD-1) or its associated ligand (PD-L-1) are targeted by the inhibitors. ${ }^{6,7}$ The immune system can be manipulated at two different stages, as

Authors: ${ }^{\mathrm{A}}$ internal medicine trainee, Velindre Cancer Centre, Cardiff, UK; ${ }^{B}$ specialty registrar in diabetes and endocrinology, University Hospital of Wales, Cardiff, UK; ${ }^{C}$ consultant endocrinologist, University Hospital of Wales, Cardiff, UK
CTLA- 4 acts in the early stage of triggering an antigen response, while PD-1 and PD-L-1 act in modulating the interaction with peripheral tissue. ${ }^{8}$ For this reason, combination therapy has been demonstrated to be effective. ${ }^{5,8}$ The mechanisms of action are summarised in Fig 1.

Ipilimumab, a CTLA-4 inhibitor, became the first approved checkpoint inhibitor in 2011, with 1-2-year survival rates doubling for metastatic melanoma, including for patients with advanced disease. ${ }^{7}$ By 2014, the PD-1 inhibitors nivolumab and pembrolizumab were approved, showing superior and sustained increases in melanoma survival. ${ }^{8}$ PD-1 inhibitors and newer PD-L-1 inhibitors are now licensed to treat a variety of other cancers, including renal cell carcinoma and non-small cell lung cancer (Table 1). ${ }^{9,10}$ Combination of CTLA-4 and PD-1 inhibitors has been

a

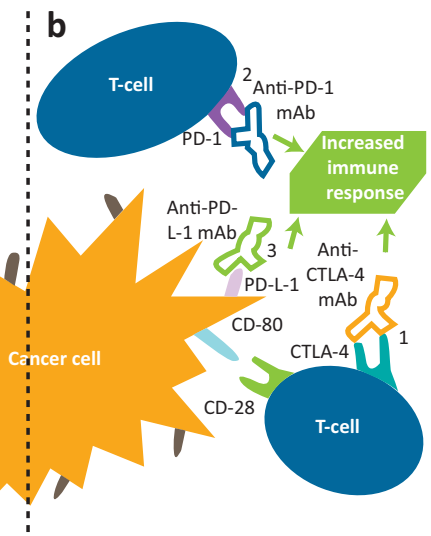

Fig 1. Simplified illustration of the mechanism of checkpoint inhibitor modulation of the immune response to cancer cells. a) Normal physiological state. In the unaltered physiological state, detection of cancer cell antigens, either via antigen-presenting cells or cancer cells themselves, causes T-cell activation. Pictured are two physiological mechanisms that reduce exaggerated immune response, but also immunosurveillance and therefore elimination of cancer cells. (1) CTLA-4 outcompetes CD-28 to bind to CD-80. Instead of further activation by CD-28, CTLA-4 sends inhibitory signals to deactivate T-cells. (2) Binding of PD-L-1 on the cancer cell or antigen-presenting cell to PD-1 on a T-cell activates inhibitory signals to immune response and encourages apoptosis of immune cells. b) State with checkpoint inhibitor therapy. Checkpoint inhibitor therapy acts on both mechanisms via three targets. (1) Anti-CTLA-4 mAb binds to CTLA-4, resulting in subsequent promotion of T-cell activation and survival by the CD-28 pathway. (2) Anti-PD-1 mAb binds to PD-1 on T-cells, preventing a negative feedback pathway. (3) Anti-PD-L-1 mAb binds to PD-L-1 on cancer cells, preventing the same negative feedback. CTLA-4 $=$ cytotoxic $\mathrm{T}$-lymphocyte antigen $4 ; \mathrm{mAb}=$ monoclonal antibody; PD-1 = protein death 1; PD-L-1 = protein death ligand 1. 


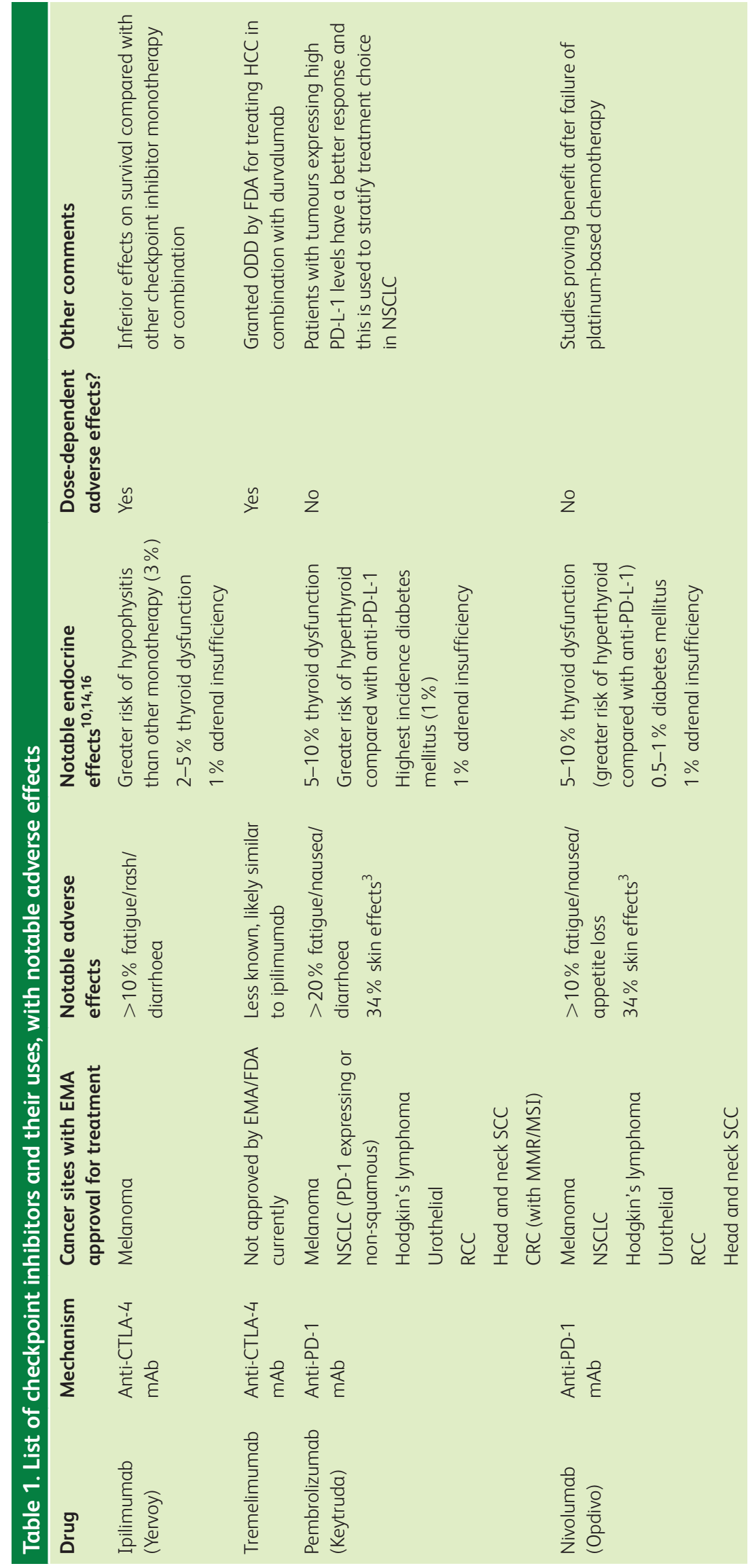




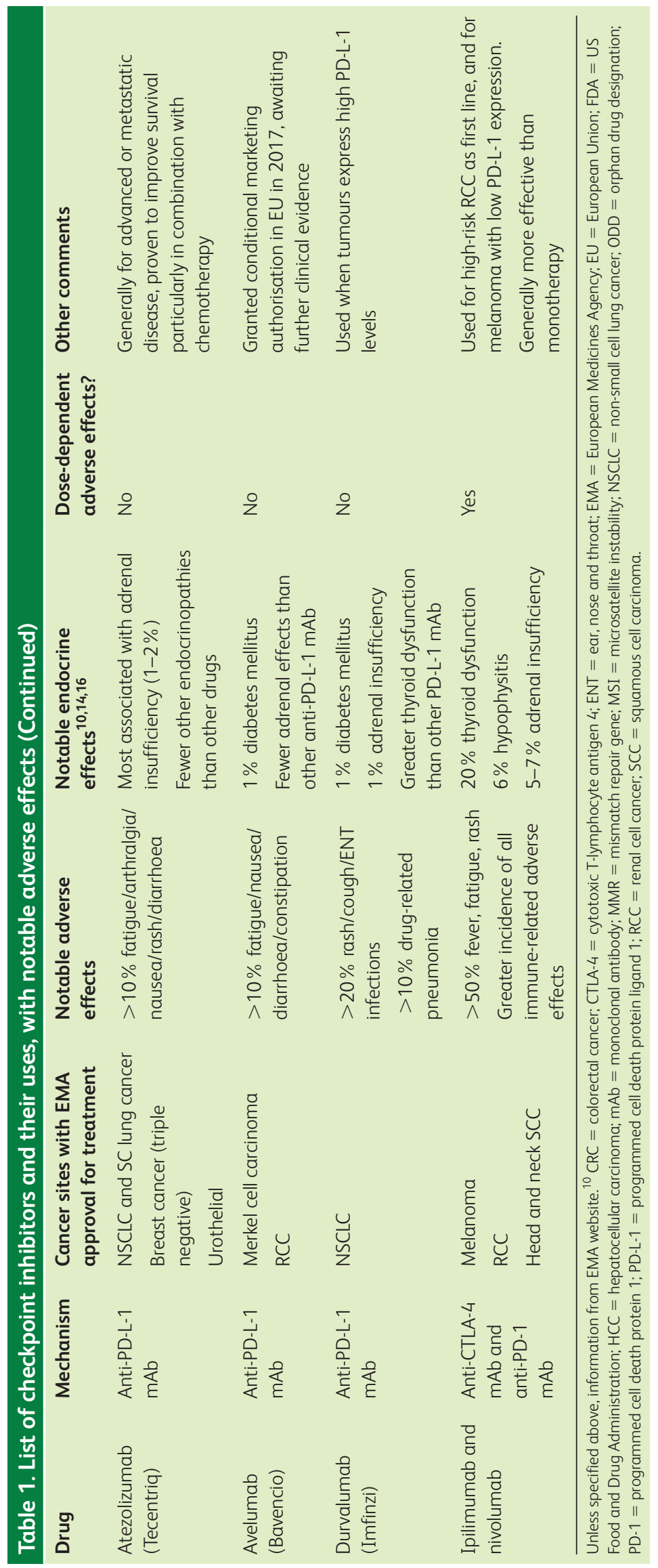


shown to be effective in metastatic melanoma, colorectal and renal cancer, with a $58 \% 3$-year survival for metastatic melanoma patients (which had previously been $20 \%$ ). ${ }^{9}$ These survival rates demonstrate that these immunotherapy treatments have thus revolutionised the treatment of certain malignancies with a historically poor prognosis.

Despite these encouraging survival data, the drugs are not without adverse effects, ranging from mild biochemical abnormalities to fatal events (incidence reported as $0.64 \%$ ). ${ }^{4}$ The mechanism is due to the exaggerated immune response towards non-cancerous cells, causing significant inflammation and destruction. ${ }^{11}$ The most commonly affected systems include the skin, gastrointestinal system, endocrine system and liver. ${ }^{2,12}$ The National Cancer Institute produced the Common Terminology Criteria for Adverse Events (CTCAE) to categorise the spectrum of toxicities from mild (grade 1) to death (grade 5), with severe symptoms requiring hospitalisation defined as grade 3 and above. ${ }^{13}$ Incidence of severe events is reported as 26\% for monotherapy and increases to $55 \%$ with combination therapy. 3,14

Immune-related endocrinopathies affect around $10 \%$ of all patients treated with checkpoint inhibitors. ${ }^{15}$ In those receiving PD-1 and CTLA-4 inhibitors, thyroid disease and hypophysitis are the most commonly encountered. ${ }^{16}$ Effects on the adrenal glands, pancreas and parathyroid glands have also been reported (Table 2). Most endocrinopathies present with non-specific symptoms, posing a diagnostic challenge as this patient group also can experience vague symptoms from malignant disease and anti-cancer treatment. The most commonly reported side effect of checkpoint inhibitors is fatigue, mostly without endocrine cause. Because of this, symptoms can dangerously be dismissed or attributed to other, less treatable causes. Diagnosis is also complicated by extensive use of corticosteroids, anti-emetics (co-administered with checkpoint inhibitors) and episodes of severe illness secondary to immunosuppression, which complicates diagnostic testing of hormonal axes.

While it is not fully understood why endocrine tissue is particularly vulnerable, hypotheses have been proposed. These include the expression of CTLA- 4 in pituitary tissue and the role of PD-1/PD-L-1 in immune tolerance disruption in the pathogenesis of autoimmune endocrinopathy. Additionally, endocrine tissue is non-regenerative and very low volume, so an immune destruction has large consequences on essential hormone secretion. Given the increasing use of these therapies, many such patients may present on the unselected medical intake. UK guidelines from the Society for Endocrinology provide practical guidance for this setting, but these are only useful if the general physician considers this differential diagnosis. ${ }^{17}$ Awareness should be raised as to the risk of such effects in patients on these medications among all physicians in order to avoid preventable morbidity and mortality.

\section{Endocrinopathies}

\section{Thyroid}

\section{Incidence and aetiology}

Primary thyroid disease represents the most common endocrine immune-related adverse effect. ${ }^{4,15,16}$ Overall incidence is reported as $8 \%$ for primary hypothyroidism and $3 \%$ for primary hyperthyroidism. ${ }^{15,16}$ Thyroid abnormalities are more commonly associated with PD-1 inhibitors and typically occur 4-10 weeks after initiation of treatment, but can occur up to 3 years later. ${ }^{16,18}$
Typically the disease process is a thyroiditis, which includes a brief, self-limiting thyrotoxic phase that lasts $3-6$ weeks, followed by a prolonged hypothyroid phase. ${ }^{19,20}$ This phase is irreversible in twothirds of patients. ${ }^{16}$ The most common mechanism described in the literature is an inflammatory destruction of the thyroid gland by cytotoxic T-cells, causing either excess thyroid hormones due to thyroid tissue breakdown, or inadequate hormone synthesis. ${ }^{15}$ Rarely, isolated hyperthyroidism or hypothyroidism may be seen, and CTLA-4 or PD-1 inhibitors can cause an autoimmunemediated Graves' disease.

\section{Clinical presentation}

Presenting symptoms mirror those outside the use of immunotherapy and include lethargy, weight gain, dry skin and constipation in hypothyroidism, and weight loss, palpitations, anxiety and sweating in hyperthyroidism. ${ }^{16,18}$ Patients may also be identified on routine screening with few or no symptoms. Severe, life-threatening illness, such as myxoedema coma or thyrotoxic storm, is rare, occurring in $0.1 \%$ of cases. ${ }^{15,18}$

\section{Diagnosis}

Diagnosis is confirmed using routine thyroid function tests (Fig 2). Autoantibodies should be tested if a diagnosis of Graves' disease is considered (usually in the presence of ophthalmopathy, persistent thyrotoxicosis or a goitre). ${ }^{17,18,21}$

\section{Treatment}

Those patients who develop mild or self-limiting thyroid disease do not require treatment and can be managed by the oncology team. They require little to no disruption to their immunotherapy treatment and simply require monitoring blood tests. Those with symptoms or severe biochemical abnormalities should be discussed with an endocrinologist and treatment initiated promptly. In such patients, immunotherapy may need to be held. ${ }^{17,18,22}$

Treatment guidelines are summarised in Fig 2. In patients with hypothyroidism, adrenal insufficiency must be excluded (or, if identified, treated) prior to commencing thyroid hormone replacement in order to prevent exacerbating an adrenal crisis. ${ }^{17,18,23}$ Levothyroxine should be titrated according to thyroid-stimulating hormone (TSH) in primary hypothyroidism, and thyroxine (T4) levels in secondary hypothyroidism. For those with hyperthyroidism, symptomatic treatment is often adequate as many self-resolve. ${ }^{16,22}$ Blockade of thyroid hormone synthesis is ineffective for inflammatory hyperthyroid disease, so unless there is convincing evidence of Graves' disease, it is not recommended. ${ }^{17,18}$

\section{Pituitary}

\section{Incidence and aetiology}

Hypophysitis is the most common endocrinopathy caused by CTLA- 4 inhibitors. ${ }^{24}$ It refers to inflammation of the pituitary gland or stalk, causing disruption of the hypothalamic-pituitary axis. Incidence is reported as $1.3 \%$ overall, with incidence on CTLA- 4 inhibitors and combination therapy reported as 3\% and $6 \%$ respectively. ${ }^{15}$ Disruption of the hypothalamic-pituitary axis occurs due to pituitary damage, likely secondary to complement-, antibody- and lymphocyte-driven processes, with pituitary necrosis occurring in severe disease. ${ }^{25}$ Hypophysitis can typically 


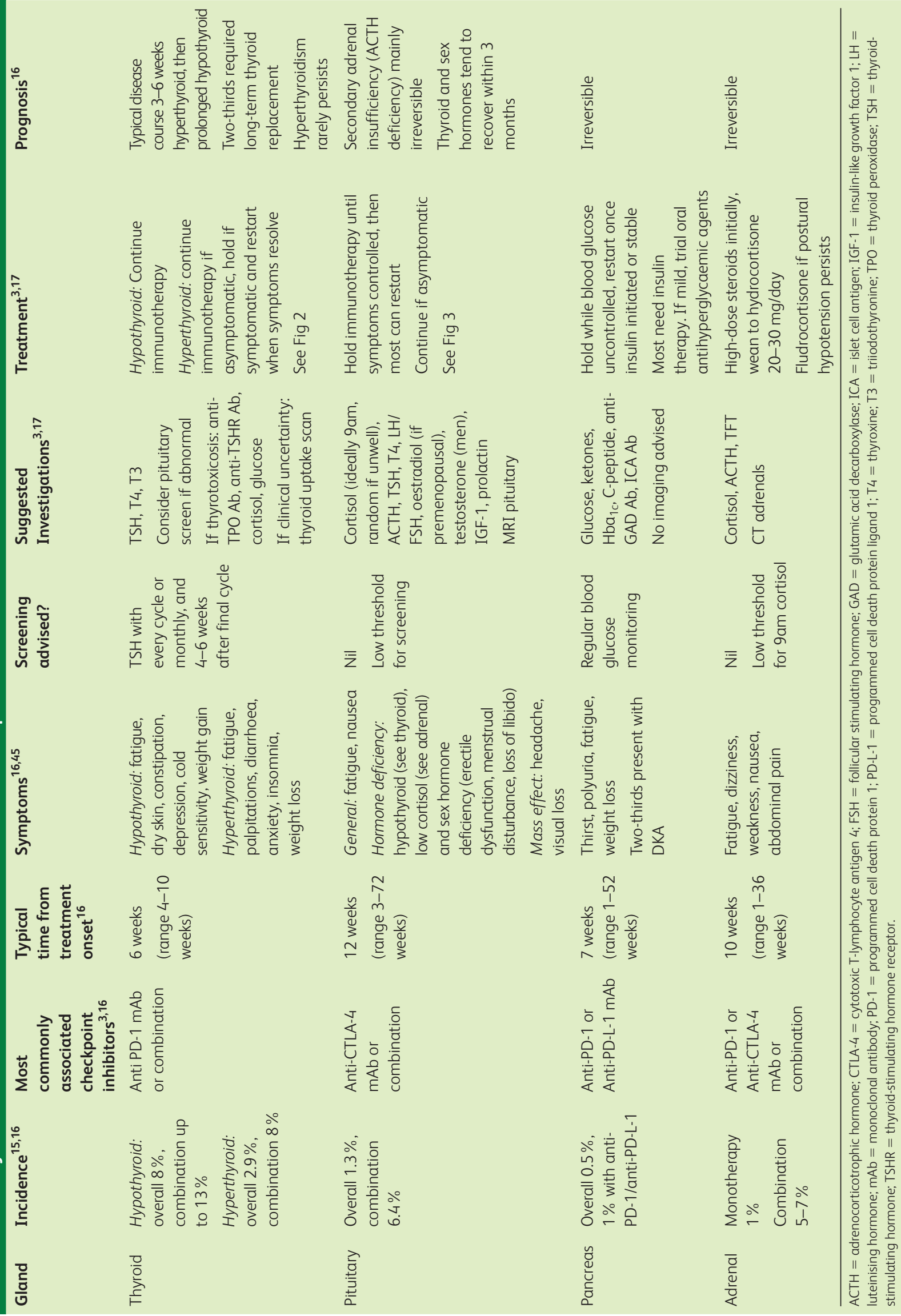




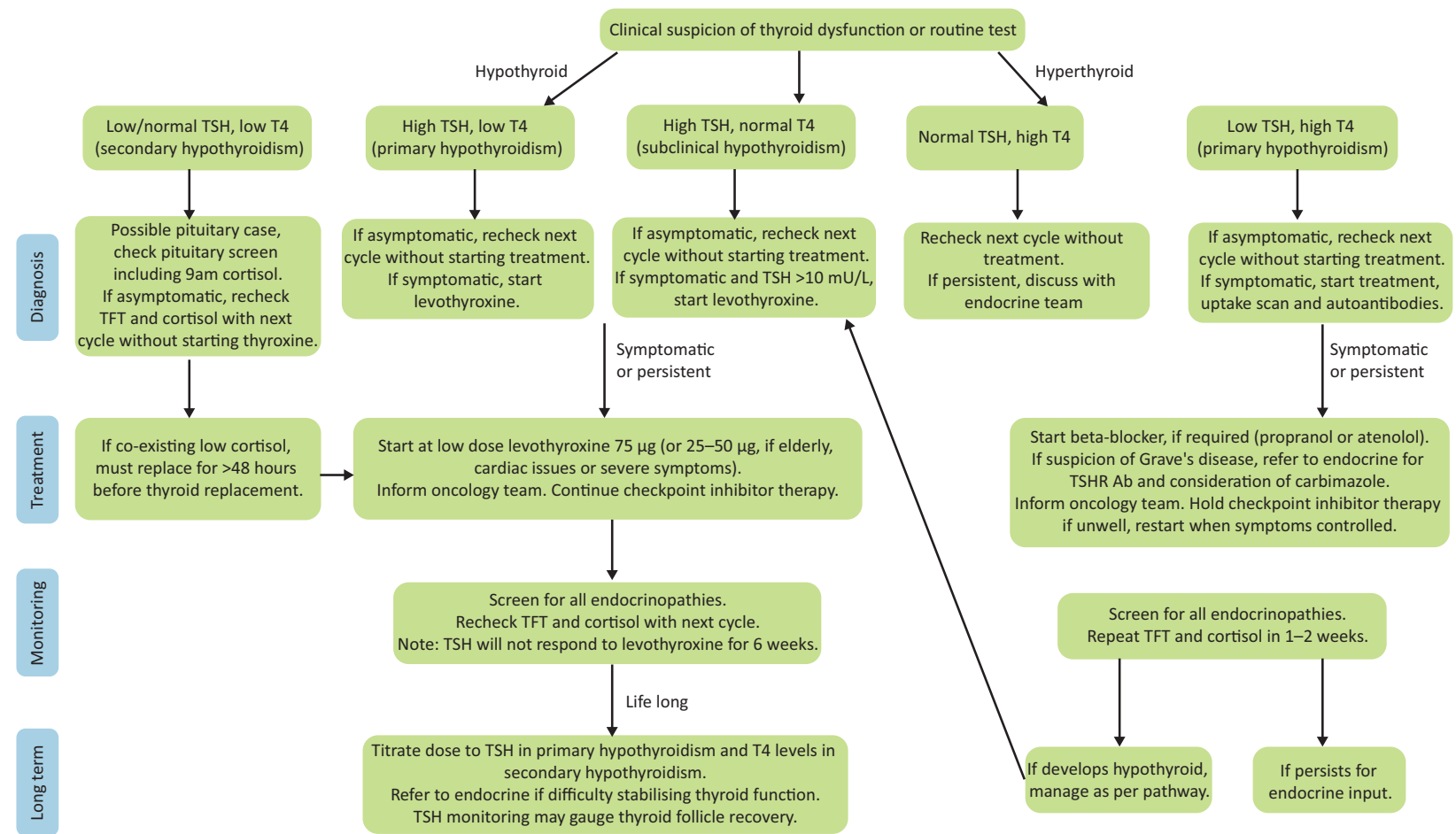

Fig 2. Management of thyroid dysfunction secondary to checkpoint inhibitors: a clinical guide. Information as per Society for Endocrinology guidelines and UK Oncology Nursing Society. ${ }^{17}{ }^{45} \mathrm{Ab}=$ antibody; T4 = thyroxine; TFT = thyroid function test; TSH = thyroid-stimulating hormone; TSHR = thyroidstimulating hormone receptor.

present approximately 8-10 weeks following initiation of therapy, less commonly after the first 3 months, although presentation many months later can be seen. ${ }^{16}$ The incidences of pituitary hormone deficiencies are reported as $83 \%$ for secondary adrenal deficiency, $77 \%$ for secondary hypothyroidism and $53 \%$ for hypogonadotrophic hypogonadism. ${ }^{18,19,26}$ It is common for patients to exhibit multiple deficiencies, with three being the most commonly reported number. ${ }^{25}$

\section{Clinical pres entation}

Presentation can be non-specific, including symptoms of nausea, fatigue and headache, many of which are common in cancer patients and often associated with its treatment. At presentation, $60 \%$ of patients have a headache. ${ }^{25}$ Other symptoms relate to secondary hormone deficiency, with $72 \%$ of patients exhibiting symptoms of secondary cortisol deficiency at presentation (dizziness, fatigue, nausea, vomiting, abdominal pain). ${ }^{25}$ Posterior lobe disorders are rare; in those who do experience this, symptoms mainly relate to secondary hormone deficiencies. ${ }^{18}$ Visual disturbance is rare in comparison to primary pituitary disorders as minimal pituitary enlargement occurs, thus reducing the risk of optic chiasm involvement. ${ }^{25}$

\section{Diagnosis}

Diagnosis includes biochemical assessment of the hypothalamicpituitary axis and imaging of the pituitary. If hypophysitis is suspected, a full pituitary screen is recommended, as summarised in Fig 3.

Imaging of the pituitary is pertinent in ruling out metastatic disease, with magnetic resonance imaging (MRI) being the gold standard. ${ }^{3,17,21}$ Society for Endocrinology guidelines recommend that this is done on an urgent basis, particularly if headache, diplopia or cranial nerve palsies are present. ${ }^{17}$ The pituitary may show mild to moderate enlargement in the acute phase and possible stalk thickening. ${ }^{27}$ Resolution of changes is often seen on repeat imaging within days to weeks. ${ }^{2,27}$

\section{Treatment}

Management involves hormone replacement and referral to an endocrinologist. Those patients requiring glucocorticoid replacement usually require this for the long term. As previously mentioned, glucocorticoid should be initiated prior to thyroid replacement to avoid precipitating an adrenal crisis. Physiological doses are advised, with no evidence that high-dose steroid improves survival or reversibility. ${ }^{17,27}$ Intravenous methylprednisolone is only recommended for neurological complications of hypophysitis. ${ }^{17}$ Maintenance of $5 \mathrm{mg}$ prednisolone once daily or hydrocortisone 10 $\mathrm{mg}$ twice daily is generally sufficient. Patient education is important, particularly regarding sick day rules and parenteral hydrocortisone administration if severely unwell. ${ }^{23}$ Mineralocorticoids do not need to be replaced as they are still produced due to renin-angiotensin regulation. $^{3,15}$

In patients requiring thyroid and gonadal hormone replacement, this is usually on a temporary basis. Thyroid replacement should start at a low dose and be titrated to T4 levels, with TSH monitoring useful for indication of pituitary thyrotroph recovery. ${ }^{3}$ Sex hormone replacement can be considered with oestradiol for premenopausal women and testosterone for men, with caution paid to contraindications - particularly malignancy related and risk of thrombosis with oestrogen replacement. ${ }^{29}$ 


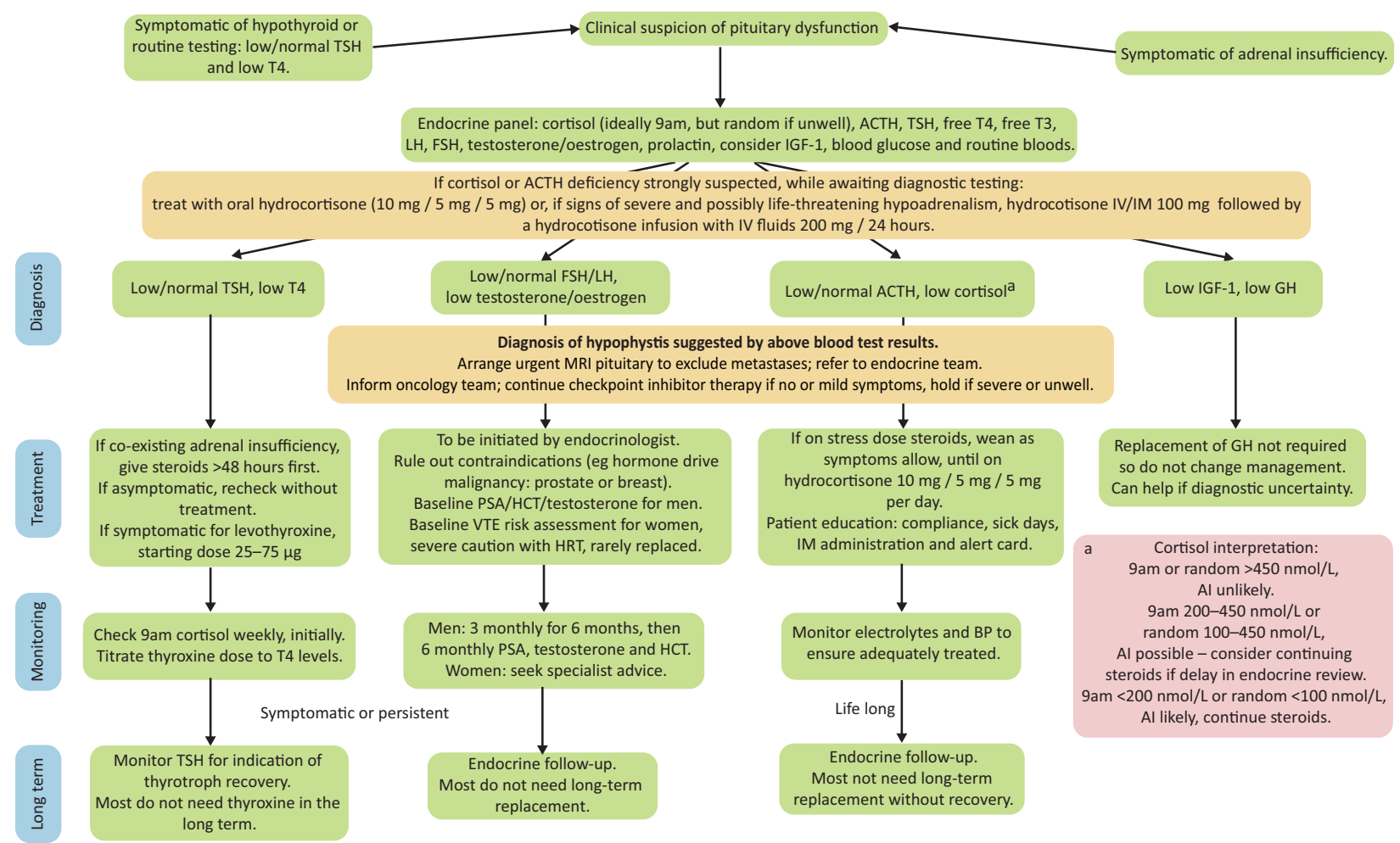

Fig 3. Management of hypophysitis secondary to checkpoint inhibitors: a clinical guide. Information as per Society for Endocrinology guidelines and UK Oncology Nursing Society. ${ }^{17,45}$ Ab = antibody; ACTH = adrenocorticotrophic hormone; AI = adrenal insufficiency; BP = blood pressure; FSH = follicular stimulating hormone; $\mathrm{GH}=$ growth hormone; $\mathrm{HCT}=$ haematocrit; IGF-1 = insulin-like growth factor 1; IM = intramuscular; IV = intravenous; $\mathrm{LH}=$ luteinising hormone; PSA = prostate specific antigen; T4 = thyroxine; $\mathrm{T} 3$ = triiodothyronine; TFT = thyroid function test; $\mathrm{TSH}=$ thyroid-stimulating hormone; TSHR $=$ thyroid-stimulating hormone receptor.

\section{Pancreas}

\section{Incidence and aetiology}

While less common than thyroid or pituitary disease, the lifethreatening consequences of pancreatic tissue destruction caused by checkpoint inhibitors are clinically important. PD-1 and PD-L-1 agents are associated with a $1 \%$ incidence of diabetes mellitus (DM), either new-onset type $1 \mathrm{DM}$ or worsening type $2 \mathrm{DM}$. $^{16,30}$ The mechanism appears similar to the permanent autoimmune process of type 1 DM, but with faster, aggressive destruction of beta islet cells, likely mediated by T-cells, requiring rapid insulin replacement. ${ }^{30,31}$ Of those developing type 1 DM secondary to immunotherapy, approximately $70 \%$ of patients exhibit a recognised genetic predisposition to $\mathrm{DM}^{32}$ The alternative cause of corticosteroid use should also be considered as a more common cause for hyperglycaemia.

\section{Clinical presentation}

Presenting symptoms include thirst, polyuria, polydipsia, dehydration, weight loss and lethargy. ${ }^{16}$ Approximately two-thirds of patients present in diabetic ketoacidosis, with the remainder with severe hyperglycaemia. ${ }^{32}$ The median time from treatment initiation to presentation is $7-17$ weeks, with a small number of cases presenting years after treatment initiation. ${ }^{30,33}$

\section{Diagnosis}

Investigations tend to reveal an elevated plasma glucose, a very low or absent serum $\mathrm{C}$-peptide, reflecting the rapid destruction of pancreatic tissue, and only a mildly elevated $\mathrm{HbA}_{1}$, given the acuity of the disease process. ${ }^{16,30}$ Autoantibodies are positive in about half of patients, with cases of both pre-existing and newly developed autoantibodies both reported. ${ }^{16,19,32}$ Despite this, Society for Endocrinology guidelines state that pancreatic autoantibodies (islet cell and GAD (glutamic acid decarboxylase)) should be taken, with potential use to distinguish between type 1 and $2 \mathrm{DM}^{17}$

\section{Treatment}

Given the aggressive pathophysiology, insulin therapy is usually essential. It is noted that, unlike other immune-related adverse events, the disease process is worsened by glucocorticoids as a consequence of increased insulin resistance with no evidence to support reversal of the beta cell damage. ${ }^{30}$ Routinely, immunotherapy is held until the hyperglycaemia is controlled, but cessation of treatment is not always required. ${ }^{3,22}$ In those patients in whom new hyperglycaemia is mild (less than $11 \mathrm{mmol} / \mathrm{L}$ ) or type $2 \mathrm{DM}$ is pre-existing, treatment may continue uninterrupted, with counselling on lifestyle modification and consideration of oral antihyperglycaemic agents, with microvascular and macrovascular complication management as per the National Institute for Health and Care Excellence (NICE) guidelines for type $2 \mathrm{DM}^{3,21}$

\section{Adrenal}

\section{Incidence and aetiology}

Primary adrenal insufficiency (PAI) is reported as $1 \%$ with either CTLA-4 or PD-1 monotherapy, and 5-7\% on combination 
therapy. ${ }^{13,15}$ Its likely cause is irreversible antibody-mediated, and possible T-cell-mediated, immune destruction of the adrenal glands. ${ }^{16,34,35}$ Production of glucocorticoids is predominantly affected, with only one-third of patients symptomatic of mineralocorticoid deficiency. ${ }^{19}$

\section{Clinical presentation}

Presenting symptoms are non-specific including fatigue, nausea, dizziness and anorexia, so a high clinical index of suspicion is required. In a review of 451 patients with PAI secondary to checkpoint inhibitors, $90 \%$ presented with adrenal crisis, and mortality was recorded as $7 \% .{ }^{34}$ Time of onset can range from 6 days to 18 months post-treatment initiation. ${ }^{34}$

A thorough history of recent steroid use is needed, including inhaled, topical or injected therapy. All can suppress adrenal function and adrenal insufficiency from exogenous steroid use is a more common diagnosis than adrenalitis.

\section{Diagnosis}

Electrolyte monitoring may aid diagnostic suspicion and should also be monitored following diagnosis, as hyponatraemia and hyperkalaemia can occur. Diagnosis can be made from a low morning cortisol with a high morning adrenocorticotropic hormone (ACTH), with confirmation provided by a poor response to synthetic $\mathrm{ACTH} .{ }^{21}$ Computed tomography (CT) of the adrenal glands may demonstrate atrophy or adrenalitis and facilitate exclusion of other causes. Anti-21-hydroxylase antibodies may also be helpful in excluding autoimmune aetiology, but are not commonly done. ${ }^{17,21,34}$

\section{Treatment}

Management requires prompt replacement of glucocorticoids (hydrocortisone $20-30 \mathrm{mg} /$ day), and mineralocorticoids (fludrocortisone) if required. If the patient is acutely or life-threateningly unwell initially, Society for Endocrinology guidelines advocate higher-dose glucocorticoids, tapering to physiological dose following clinical recovery, with prompt endocrine referral..$^{17}$ As with pituitaryrelated insufficiency, patient education in terms of sick day rules and emergency hydrocortisone is vital. Patients can be supplied with an NHS steroid card to improve management in the emergency setting. ${ }^{36}$ It is advised to hold immunotherapy while unwell, with scope for continuing it later when stable on hormone replacement. ${ }^{21,22}$

\section{Parathyroid}

\section{Incidence and aetiology}

Parathyroid involvement appears to be exceedingly rare, with only five case reports of hypoparathyroidism reported and no hyperparathyroidism. ${ }^{37-41}$ Unlike other endocrinopathies, most patients have described a reversible inflammatory process. ${ }^{39}$ Onset is later, reported between 4 and 11 months following initiation of either PD-1 inhibitor or combination therapy. ${ }^{39}$

\section{Clinical presentation}

Hypocalcaemia can present as nausea, vomiting, paraesthesia, cramps, abdominal pain, confusion and fatigue. ${ }^{40,42}$ If severe, lifethreatening arrhythmias or seizures can occur.

\section{Diagnosis}

Diagnosis is confirmed by a low serum calcium with a low or non-elevated parathyroid hormone level. Serum magnesium, phosphate and vitamin D should be monitored alongside calcium. Ruling out alternative causes for hypoparathyroidism, such as previous surgery, should be included in the work-up. Electrocardiography should be performed to assess the QT interval.

\section{Treatment}

Management is of calcium replacement, either intravenously or orally depending on the severity of deficiency. Cardiac monitoring may be needed. Ongoing management is then with the initiation and titration of alfacalcidol according to serum calcium levels. There is insufficient evidence to dictate whether cessation of immunotherapy is necessary, and this should therefore be decided on a case-by-case basis.

In addition, when considering calcium disorders, the relevance of other oncology factors must be taken into consideration, such as the presence of skeletal metastases and drugs relevant to their management, such as the use of bisphosphonates or denosumab. Hypocalcaemia is an important side effect of these drugs.

\section{Other rare endocrinopathies}

\section{Diabetes insipidus}

Diabetes insipidus (DI) is rarely reported in immunotherapy - only two cases to date - in comparison to pituitary metastasis as a cause. It is thought to be a consequence of inadequate antidiuretic hormone $(A D H)$ secretion secondary to hypophysitis affecting the posterior pituitary. ${ }^{26,43}$ Patients presented with polyuria, polydipsia, thirst and fatigue, with diagnosis being confirmed by serum osmolality $>295 \mathrm{mmol} / \mathrm{kg}$ and urine osmolality $<600 \mathrm{mmol} / \mathrm{kg}$. Spontaneous remission was demonstrated in both cases, with one requiring no treatment and the other demonstrating reversibility after 6 weeks of desmopressin (DDAVP). ${ }^{26}$ It is important to note that there is potential for DI to be masked until concomitant ACTH deficiency is treated.

Furthermore, only one case of ACTH-dependent Cushing's syndrome has been reported. ${ }^{19,44}$ Presentation included mild symptoms of cortisol excess (weight gain, depression and fatigue) and there was spontaneous resolution, then development of secondary cortisol insufficiency. By far a more common cause is iatrogenic Cushing's syndrome, with corticosteroids used frequently for cancer-related symptoms, non-endocrine immunerelated adverse events and hypersensitivity reactions.

Polyglandular endocrinopathies have also been described, commonly the thyroid gland and one of the other aforementioned endocrinopathies. ${ }^{19}$

\section{Other considerations}

\section{Clinician and patient education}

Endocrine complications of checkpoint inhibitors require prompt diagnosis in order to prevent significant morbidity and mortality. Patient education of these adverse effects is important to aid early recognition of the endocrinopathies and prevent delay in diagnosis. Patients should be counselled to inform healthcare professionals if they are currently or previously receiving checkpoint inhibitors. Dissemination of information to physicians in both primary and secondary care is also pertinent in supporting prompt diagnoses. ${ }^{45}$

\section{Screening}

Since the presentation of these complications may be indolent and non-specific, screening offers a potentially useful tool. Regular 
monitoring of thyroid function and blood glucose is universally recommended, but screening for adrenal and pituitary disease is more challenging. ${ }^{3,21}$ Although the consequences of glucocorticoid deficiency carry high morbidity and mortality, and thus screening may appear sensible, this is a topic of debate. No national guidance is available regarding UK endocrinopathy screening. In terms of being able to identify individuals at increased risk of developing complications, early work suggests that genetic predispositions may play a role in this. ${ }^{9}$

Fluorodeoxyglucose-positron emission tomography (FDG-PET) scans can also incidentally diagnose immunotherapy-mediated endocrinopathies by diffuse uptake. Comparison with baseline scans is required and often only patients with curative intent will receive regular PET scans, which doesn't typically align with those receiving immunotherapy currently. Patients may not exhibit symptoms at this point, which poses a therapeutic dilemma.

\section{The role of high-dose steroids}

There is debate as to whether high-dose steroids may be helpful in the management of checkpoint-induced endocrinopathies. Current UK Society for Endocrinology guidelines advise that there is no indication for intravenous methylprednisolone for routine management of endocrinopathies, with hypophysitis with associated neurological features being the only exception. ${ }^{17}$ In contrast, European guidelines recommend use in severe hypophysitis and severe thyrotoxicosis or thyroiditis; however, there have not been proven benefits for milder disease or other endocrinopathies. ${ }^{3}$ Limited evidence is available to support these guidelines, but benefit likely outweighs risks when pituitary oedema is causing severe headache, cranial nerve palsy or visual disturbance. $^{29}$

Immunotherapy itself is not the only endocrine consideration in cancer treatment. While taking checkpoint inhibitors, one-third of patients take corticosteroids, mainly to treat non-endocrine adverse effects or for brain metastases. ${ }^{46}$ Awareness of detrimental effects of steroids, such as steroidinduced hyperglycaemia, iatrogenic adrenal insufficiency or immunosuppression, should also be considered. ${ }^{30}$

\section{Adverse effects and cancer prognosis}

Some retrospective data have suggested that patients who develop immunotherapy-induced hypophysitis survive on average 1 year longer than those who do not, and those who develop thyroid dysfunction survive 2 years longer than those who do not. ${ }^{18}$ However, the population sizes in these studies are small, and data on long-term outcomes of endocrine effects may be skewed by follow-up bias. Early evidence has suggested that timing of adverse effects may be associated with survival, reporting that developing the effects within the first 3 months of treatment is associated with better outcomes. Further work is needed before firm conclusions can be made. ${ }^{47}$

\section{COVID-19}

The COVID-19 pandemic has brought additional challenges to cancer therapy. Early outcomes suggest that cancer patients are at increased risk of mortality and morbidity, but there is currently no evidence that use of checkpoint inhibitors worsens outcomes. ${ }^{48,49}$ Conflicting hypotheses exist suggesting that checkpoint inhibitors could either dampen the viral response by exhaustion of T-cells, or opposingly, amplify the exaggerated immune response to viruses like SARS-CoV-2. ${ }^{48,50}$ Experience from previous viruses suggests that the latter rarely occurs. To reduce the risk in vulnerable patients, regular screening for COVID-19 plus prompt recognition and management of any immune-related adverse effects is recommended.

\section{Conclusion}

Checkpoint inhibitors offer huge benefits for those with advanced cancer and although adverse effects are significant, the increase in survival that these treatments offer justifies this risk. Prompt identification and management of endocrine effects can minimise impact on patient experience and mortality. Further research is needed to improve understanding of predisposing and protective factors, and ways of improving current management.

\section{Key points}

> Endocrine adverse effects of checkpoint inhibitor immunotherapy occur in around $10 \%$ of patients.

> Thyroid disease and hypophysitis are the most common, which can occur from weeks to many months after treatment.

> Primary adrenal insufficiency and diabetes mellitus are also reported, along with other more rare endocrinopathies.

$>$ Early recognition and management are key, with referral to an endocrinologist for ongoing management.

$>$ Non-specific symptoms require a high index of suspicion for investigation.

$>$ Patient and clinician education and awareness of these effects are vital.

> Some debate remains surrounding the use of screening for endocrinopathies and high-dose steroids in their treatment.

> Early data suggest that endocrine adverse effects may be associated with better cancer survival outcomes.

\section{References}

1 Martins F, Sofiya L, Sykiotis GP et al. Adverse effects of immunecheckpoint inhibitors: epidemiology, management and surveillance. Nat Rev Clin Oncol 2019;16:563-80.

2 Del Rivero J, Cordes LM, Klubo-Gwiezdzinska J, Madan RA, Nieman LK, Gulley JL. Endocrine-related adverse events related to immune checkpoint inhibitors: proposed algorithms for management. Oncologist 2020;25:290-300.

3 Haanen JBAG, Carbonnel F, Robert $C$ et al. Management of toxicities from immunotherapy: ESMO Clinical Practice Guidelines for diagnosis, treatment and follow-up. Ann Oncol 2018;29(Suppl 4): iv264-6.

4 De Velasco G, Je Y, Bossé D et al. Comprehensive meta-analysis of key immune-related adverse events from CTLA-4 and PD-1/PD-L1 inhibitors in cancer patients. Cancer Immunol Res 2017;5:312-8.

5 Castinetti $F$, Borson-Chazot F. Introduction to expert opinion on endocrine complications of new anticancer therapies. Ann Endocrinol (Paris) 2018;79:535-8.

6 Byun DJ, Wolchok JD, Rosenberg LM, Girotra M. Cancer immunotherapy - immune checkpoint blockade and associated endocrinopathies. Nat Rev Endocrinol 2017;13:195-207.

7 Lugowska I, Teterycz P, Rutkowski P. Immunotherapy of melanoma. Contemp Oncol (Pozn) 2018;22:61-7.

8 González-Rodríguez E, Rodríguez-Abreu D, Spanish Group for Cancer Immuno-Biotherapy (GETICA). Immune checkpoint inhibitors: review and management of endocrine adverse events. Oncologist 2016;21:804-16. 
9 Fares CM, Van Allen EM, Drake CG, Allison JP, Hu-Lieskovan S. Mechanisms of resistance to immune checkpoint blockade: why does checkpoint inhibitor immunotherapy not work for all patients? Am Soc Clin Oncol Educ Book 2019;39:147-64.

10 European MedicinesAgency. European public assessment reports. EMA, 2020. www.ema.europa.eu/en/medicines [Accessed 09 February 2021].

11 Seebacher NA, Stacy AE, Porter GM, Merlot AM. Clinical development of targeted and immune based anti-cancer therapies. J Exp Clin Cancer Res 2019;38:156.

12 Khoja L, Day D, Wei-Wu Chen T, Siu LL, Hansen AR. Tumour- and class-specific patterns of immune-related adverse events of immune checkpoint inhibitors: a systematic review. Ann Oncol 2017;28:2377-85.

13 US Department of Health and Human Sciences. Common Terminology Criteria for Adverse Events (CTCAE) Version 5.0, 2017. https://ctep.cancer.gov/protocolDevelopment/electronic applications/docs/CTCAE_v5_Quick_Reference_8.5x11.pdf [Accessed 09 February 2021].

14 Cukier P, Santini FC, Scaranti M, Hoff AO. Endocrine side effects of cancer immunotherapy. Endocr Relat Cancer 2017;24:T331-47.

15 Barroso-Sousa R, Barry WT, Garrido-Castro AC et al. Incidence of endocrine dysfunction following the use of different immune checkpoint inhibitor regimens: a systematic review and metaanalysis. JAMA Oncol 2018;4:173-82.

16 de Filette J, Andreescu CE, Cools F, Bravenboer B, Velkeniers B. A systematic review and meta-analysis of endocrine-related adverse events associated with immune checkpoint inhibitors. Horm Metab Res 2019;51:145-56.

17 Higham CE, Olsson-Brown A, Carroll P et al. Society for Endocrinology Endocrine Emergency Guidance: Acute management of the endocrine complications of checkpoint inhibitor therapy. Endocr Connect 2018;7:G1-7.

18 Chang LS, Barroso-Sousa R, Tolaney SM et al. Endocrine toxicity of cancer immunotherapy targeting immune checkpoints. Endocr Rev 2019;40:17-65.

19 Tan MH, Iyengar R, Mizokami-Stout K et al. Spectrum of immune checkpoint inhibitors-induced endocrinopathies in cancer patients: a scoping review of case reports. Clin Diabetes Endocrinol 2019;5:1.

20 Brancatella A, Viola N, Brogioni S et al. Graves' disease induced by immune checkpoint inhibitors: a case report and review of the literature. Eur Thyroid J 2019;8:192-5.

21 Brahmer JR, Lacchetti C, Schneider B] et al. Management of immune-related adverse events in patients treated with immune checkpoint inhibitor therapy: American Society of Clinical Oncology Clinical Practice Guideline. J Clin Oncol 2018;36:1714-68.

22 Trinh S, Le A, Gowani S, La-Beck NM. Management of immunerelated adverse events associated with immune checkpoint inhibitor therapy: a minireview of current clinical guidelines. Asia Pac J Oncol Nurs 2019;6:154-60.

23 Vaidya B, Chakera AJ, Dick C. Addison's disease. BMJ 2009;339: b2385.

24 Albarel F, Castinetti F, Brue T. Management of endocrine disease: immune check point inhibitors-induced hypophysitis. Eur J Endocrino 2019:181:R107-18.

25 Caturegli P, Di Dalmazi G, Lombardi M et al. Hypophysitis secondary to cytotoxic T-lymphocyte-associated protein 4 blockade: insights into pathogenesis from an autopsy series. Am J Pathol 2016;186:3225-35.

26 Zhao C, Tella SH, Del Rivero ] et al. Anti-PD-L1 treatment induced central diabetes insipidus. J Clin Endocrinol Metab 2018;103: 365-9.

27 Faje AT, Sullivan R, Lawrence D et al. Ipilimumab-induced hypophysitis: a detailed longitudinal analysis in a large cohort of patients with metastatic melanoma. J Clin Endocrinol Metab 2014;99:4078-85.
28 Min L, Hodi FS, Giobbie-Hurder A et al. Systemic high-dose corticosteroid treatment does not improve the outcome of ipilimumabrelated hypophysitis: a retrospective cohort study. Clin Cancer Res 2015;21:749-55.

29 Barroso-Sousa R, Ott PA, Hodi FS et al. Endocrine dysfunction induced by immune checkpoint inhibitors: Practical recommendations for diagnosis and clinical management. Cancer 2018;124:1111-21.

30 Quandt Z, Young A, Anderson M. Immune checkpoint inhibitor diabetes mellitus: a novel form of autoimmune diabetes. Clin Exp Immunol 2020;200:131-40.

31 Kotwal A, Haddox C, Block M, Kudva YC. Immune checkpoint inhibitors: an emerging cause of insulin-dependent diabetes. BMJ Open Diabetes Res Care 2019;7:e000591.

32 Stamatouli AM, Quandt Z, Perdigoto AL et al. Collateral damage: insulin-dependent diabetes induced with checkpoint inhibitors. Diabetes 2018:67:1471-80.

33 Girotra M, Hansen A, Farooki A et al. The current understanding of the endocrine effects from immune checkpoint inhibitors and recommendations for management. JNCI Cancer Spectr 2018;2:pky021.

34 Grouthier V, Lebrun-Vignes B, Moey M et al. Immune checkpoint inhibitor-associated primary adrenal insufficiency: WHO VigiBase report analysis. Oncologist 2020;25:696-701.

35 Paepegaey AC, Lheure C, Ratour C et al. Polyendocrinopathy resulting from pembrolizumab in a patient with a malignant melanoma. J Endocr Soc 2017;1:646-9.

36 Simpson H, Tomlinson J, Wass ], Dean J, Arlt W. Guidance for the prevention and emergency management of adult patients with adrenal insufficiency. Clin Med 2020;20:371-8.

37 Trinh B, Sanchez GO, Herzig P, Läubli H. Inflammation-induced hypoparathyroidism triggered by combination immune checkpoint blockade for melanoma. J Immunother Cancer 2019;7:52.

38 Win MA, Thein KZ, Qdaisat A, Yeung SC]. Acute symptomatic hypocalcemia from immune checkpoint therapy-induced hypoparathyroidism. Am J Emerg Med 2017;35:1039.e5-7.

39 Lupi I, Brancatella A, Cetani F et al. Activating antibodies to the calcium-sensing receptor in immunotherapy-induced hypoparathyroidism. J Clin Endocrinol Metab 2020;105:dgaa092.

40 Piranavan P, Li Y, Brown E et al. Immune checkpoint inhibitor-induced hypoparathyroidism associated with calcium-sensing receptor-activating autoantibodies. J Clin Endocrinol Metab 2019;104:550-6.

41 Umeguchi $\mathrm{H}$, Takenoshita $\mathrm{H}$, Inoue $\mathrm{H}$ et al. Autoimmune-related primary hypoparathyroidism possibly induced by the administration of pembrolizumab: a case report. J Oncol Pract 2018;14:449-51.

42 Cooper MS, Gittoes NJL. Diagnosis and management of hypocalcaemia. BMJ 2008;336:1298-302.

43 Dillard T, Yedinak CG, Alumkal J, Fleseriu M. Anti-CTLA-4 antibody therapy associated autoimmune hypophysitis: serious immune related adverse events across a spectrum of cancer subtypes. Pituitary 2010;13:29-38.

44 Lupu J, Pages C, Laly P et al. Transient pituitary ACTH-dependent Cushing syndrome caused by an immune checkpoint inhibitor combination. Melanoma Res 2017;27:649-52.

45 UK Oncology Nursing Society. Acute Oncology Initial Management Guidelines. UKONS, 2018. www.ukons.org/site/assets/files/1134/ acute_oncology_initial_management_guidelines.pdf [Accessed 09 February 2021].

46 Agarwal K, Yousaf N, Morganstein D. Glucocorticoid use and complications following immune checkpoint inhibitor use in melanoma. Clin Med 2020;20:163-8.

47 Morehouse C, Abdullah SE, Gao C et al. Early incidence of immune-related adverse events (irAEs) predicts efficacy in patients (pts) with solid tumors treated with immune-checkpoint inhibitors (ICIs). J Clin Oncol 2019;37(15 suppl):2563.

48 Sullivan RJ, Johnson DB, Rini BI et al. COVID-19 and immune checkpoint inhibitors: initial considerations. J Immunother Cancer 2020;8:e000933. 
49 Pinato D], Zambelli A, Aguilar-Company ] et al. Clinical portrait of the SARS-CoV-2 epidemic in European cancer patients. Cancer Discov 2020;10:1465-74.

50 Bersanelli M. Controversies about COVID-19 and anticancer treatment with immune checkpoint inhibitors. Immunotherapy 2020;12:269-73.
Address for correspondence: Dr Andrew Lansdown,

Department of Endocrinology, University Hospital of Wales, Heath Park, Cardiff CF14 4XW, UK.

Email: andrew.lansdown@wales.nhs.uk 